\title{
Laser Ablated Carbon Thin Film from Carbon Nanotubes and Their Property Studies
}

\author{
Maheshwar Sharon ${ }^{1, \uparrow}$, M. Rusop ${ }^{2}$, T. Soga ${ }^{2}$ and Rakesh A. Afre ${ }^{2}$ \\ ${ }^{1}$ Nanotechnology Research Center, Birla College, Kalyan, 421-304, India \\ ${ }^{2}$ Dept. of Environmental Technology and Urban Planning, Nagoya Institute of Technology, Gokiso-cho, Showa-ku, Nagoya, 466-8555, Japan \\ •e-mail: sharon@iitb.ac.in \\ (Received December 4, 2007; Accepted March 10, 2008)
}

\begin{abstract}
A carbon nanotube (CNT) of diameter $\sim 20 \mathrm{~nm}$ has been synthesized by spray pyrolysis of turpentine oil using Ni/Fe catalyst. Pellet of CNTs has been used as a target to produce semiconducting carbon thin film of band gap $1.4 \mathrm{eV}$. Presence of oxygen pressure in the pulse laser deposition (PLD) chamber helped to control the $\mathrm{sp}^{3} / \mathrm{sp}^{2}$ ratio to achieve the desired band gap. Results are discussed with the help of Raman spectra, SEM TEM micrographs and optical measurements suggest that semiconducting carbon thin film deposited by PLD technique has retained its nanotubes structure except that its diameter has increased from $20 \mathrm{~nm}$ to $150 \mathrm{~nm}$.
\end{abstract}

Keywords : Semiconducting carbon, Turpentine oil for CNTs, Pulse laser deposition technique, Raman spectroscopy studies

\section{Introduction}

Chemistry of silicon and carbon being similar, scientists are developing semiconducting carbon for its various applications e.g., carbon solar cell. In some respect carbon may prove to be a better candidate for such application. Because silicon has structure based on $\mathrm{sp}^{3}$ configuration; carbon has the flexibility to form structures with $\mathrm{sp}, \mathrm{sp}^{2}$ and $\mathrm{sp}^{3}$ configurations. Thus the possibility exists with carbon to synthesize carbon materials which contains mixture of both $\mathrm{sp}^{2}$ and $\mathrm{sp}^{3}$ configuration. This is interesting because carbon materials containing $100 \% \mathrm{sp}^{2}$ is graphite with a band gap of around $0.25 \mathrm{eV}$ while carbon materials containing $100 \% \mathrm{sp}^{3}$ has diamond structure with a band gap of $5.5 \mathrm{eV}$. Carbon material containing a mixture of both $\mathrm{sp}^{2}$ and $\mathrm{sp}^{3}$ can have band gap in between $0.25 \mathrm{eV}$ to $5.5 \mathrm{eV}$. This flexible character of carbon gives a possibility to make a graded carbon solar cell covering maximum solar spectra. Sharon et al $[1,2]$ have been able to show that pyrolysis of camphor can give semiconducting carbon with band gap in the range of $0.4 \mathrm{eV}$ to $1.6 \mathrm{eV}$.

For the development of photovoltaic solar cell, it has been established theoretically that a semiconductor with a band gap of $1.4 \mathrm{eV}$ should give the highest efficiency for converting solar energy into electrical energy. Sharon et al [3-5] have been able to fabricate carbon solar cell by synthesizing semiconducting carbon from camphor of band gap $1.2 \mathrm{eV}$. They tried to dope carbon nanomaterial by burning camphor mixed with dopants (like Phosphorous or Boron) in an inert atmosphere. This doped carbon materials were used to make carbon solar cell. But problem with this approach was in controlling the concentration of doping and the band gap of the carbon nanomaterials. Therefore, it was thought to synthesize carbon nanotubes by spray pyrolysis of camphor, make its pellet and use this as a target material for getting thin film of carbon by pulse laser deposition technique. This paper gives an account of our efforts of synthesizing carbon nanotubes by spray pyrolysis and using this powder as target materials in PLD technique to get semiconducting carbon thin film with a band gap around $1.4 \mathrm{eV}$. PLD technique was selected because using this technique; thin film of carbon can be deposited from the target materials without changing its composition (i.e. ratio of $\left.\mathrm{sp}^{2} / \mathrm{sp}^{3}\right)$.

\section{Experimental}

\subsection{Preparation of CNTs}

Carbon nanotubes were synthesized by a simple and inexpensive spray pyrolysis method (Fig. 1) as reported previously [6]. These CNTs were treated with $6 \mathrm{~N} \mathrm{NaOH}$ for 3 hours and then with $6 \mathrm{~N} \mathrm{HCl}$ for 3 hours to remove any amorphous carbon and metal catalyst. Washing with distilled water was done after each treatment. Finally carbon material was dried. Pellet of this material was made with help of polyvinyl alcohol (PVA). PVA was dissolved in water such that solution was highly dense. 2-3 drops of this was added to CNT powder $(0.2 \mathrm{~g})$ and thoroughly mixed. This was used 


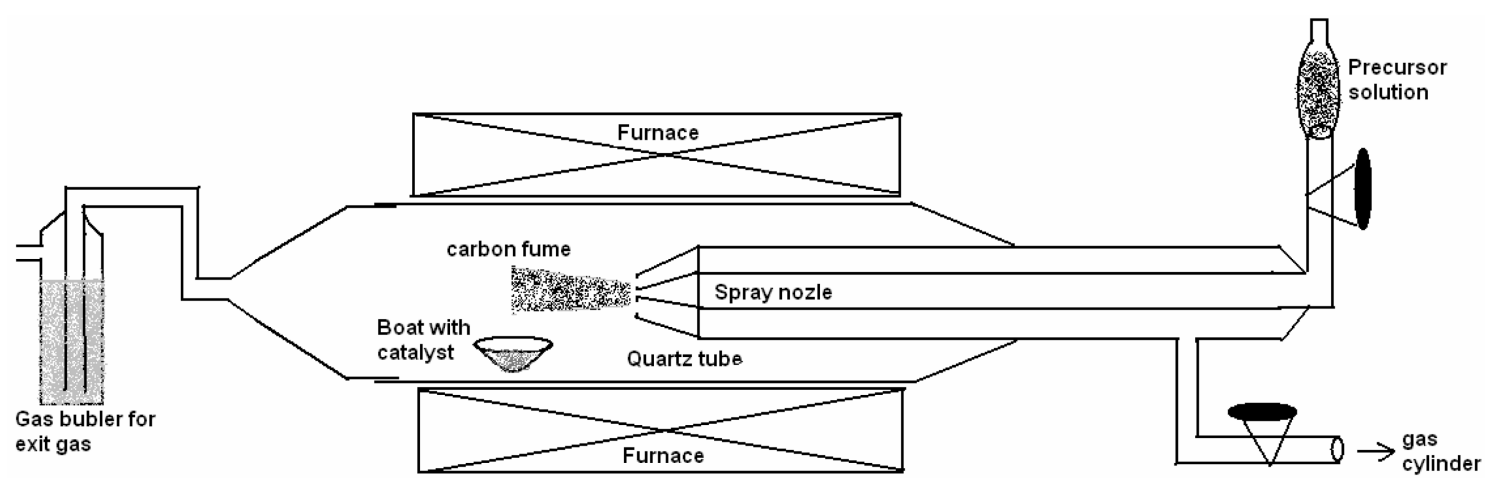

Fig. 1. Schematic representation of spray pyrolysis technique for the preparation carbon nanotubes from turpentine oil.

to make the pellet by applying pressure of $3 \mathrm{~kg} \mathrm{~cm}^{-2}$. The pellets were sintered for ten hours at $300^{\circ} \mathrm{C}$ in the nitrogen atmosphere. This pellet was used as target in the PLD unit.

\subsection{Laser ablation of carbon material}

PLD technique consists of a stainless steel vacuum chamber, which contains substrate holder attached to a substrate heater as well as a manipulator, by which distance between substrate and target is adjusted. The schematic diagram of the experimental set up is shown in Fig. 2. A

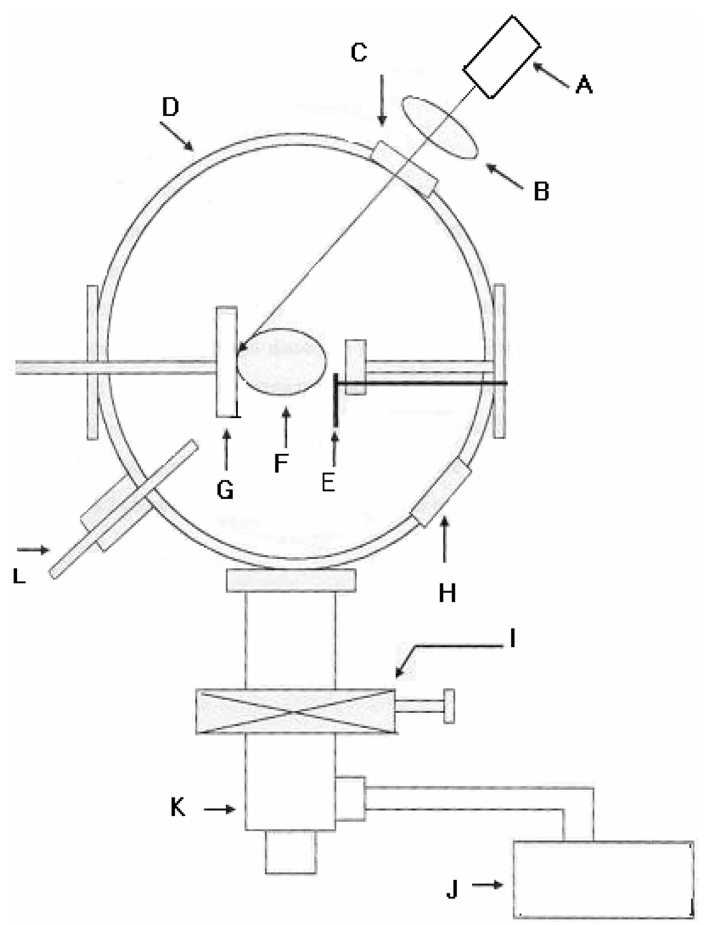

Fig. 2. A schematic diagram of the $\mathrm{XeCl}$ laser pulse deposition technique. A. Excimer laser B. Lens C. Quartz window D. Stainless chamber E. Substrate holder with heater F. Multiple target holder G. Holder connected to steper motor H. Quartz window I. Vacuum valve J. Rotary pump K. Turbo pump L. Oxygen supply.
Lumonics (Model no. PM-882, Power-9000 W, Frequency $50 \mathrm{~Hz}$ ) excimer laser has been used in this work, which can give maximum of $350 \mathrm{~mJ}$ energy having 20 pulse per sec. The laser beam was cut to a standard size by using an aperture at the laser emitting end. The aperture size was fixed at $15 \times 6 \mathrm{~cm}^{2}$. The deposition chamber lies horizontally in the same line with laser position. The laser light was focused on the target through the lens. The target holder was adjusted such that the laser light falls on it at angle of $45^{\circ}$ to the surface. The alignment of laser beam on the target was done by adjusting the manipulator. The target holder was connected to a stepper motor, by which target could be moved. An arrangements was made such that laser light falls on large area of the target.

For the pulsed laser deposition, pellet of the carbon material was mounted on the target holder with the help of silver paste. The substrate holder was attached to a heater and a temperature controller, which allows setting a desired temperature to the substrate. The chamber was connected to a rotary pump as well as a turbo molecular pump for evacuation up to $1.0 \times 10^{-5}-8.0 \times 10^{-6}$ Torr.

The quartz substrate, on which deposition was to be made, was cleaned with trichloroethylene $(97.8 \%$ pure) and acetone (AR) and then etched with $5 \% \mathrm{HNO}_{3}$ solution in Ultrasonic vibrator (UL Tasonic Cleaner; Trens - O SOLNIC) for 30 min. Then the substrates were taken out from the solution, dried and transferred to the PLD chamber for the deposition.

For the deposition of carbon thin film the chamber was first evacuated up to the maximum limit. In order to make chamber free from other gases, oxygen was flushed in several time. Finally the chamber was evacuated and oxygen of desired pressure was allowed to enter the chamber. The energy of the laser was set through the remote control according to the experimental parameters levels (Table 1). The pulse rate of laser emission was adjusted within 5 to 10 pulse/sec. The spot size of laser on the target surface and the energy of laser falling on target were measured and from these values, energy density of laser falling on the target was calculated. The rapid heating of the substrate by laser beam forms plasma towards the substrate of the material and this 
Table 1. Orthogonal L9 Table Giving the Details of Combinations of Variable Parameters Selected as per the Taguchi Optimization Methodology

\begin{tabular}{cccc}
\hline No & Laser energy (mJ) & Distance (cm) & $\begin{array}{c}\text { Partial pressure, } \\
\text { (Torr) }\end{array}$ \\
\hline 1 & 160 & 2.5 & $\mathrm{~N}_{2}$ \\
2 & 160 & 3.5 & $\mathrm{O}_{2}, 6 \times 10^{-1}$ \\
3 & 160 & 4.5 & $\mathrm{O}_{2}, 10^{-3}$ \\
4 & 190 & 2.5 & $\mathrm{O}_{2}, 6 \times 10^{-1}$ \\
5 & 190 & 3.5 & $\mathrm{O}_{2}, 10^{-3}$ \\
6 & 190 & 4.5 & $\mathrm{~N}_{2}$ \\
7 & 220 & 2.5 & $\mathrm{O}_{2}, 10^{-3}$ \\
8 & 220 & 3.5 & $\mathrm{~N}_{2}$ \\
9 & 220 & 4.5 & $\mathrm{O}_{2}, 6 \times 10^{-1}$ \\
\hline
\end{tabular}

plasma gets deposited on the surface of the substrate. This phenomenon leads to the growth of a thin film of carbon on the substrate.

Since there are several variable parameters (laser energy, distance of target from the substrate, partial pressure of oxygen, pulse rate, wave length of laser, substrate temperature, beam aperture etc) in the PLD, it was decided to take the help of Taguchi optimization methodology for finding out the most effective parameters to get carbon thin film of band gap around $1.4 \mathrm{eV}$. For this purpose, amongst the various parameters, the following parameters were fixed:

1. Wavelength of laser $308 \mathrm{~nm}$

2. No of laser shot at $5 \mathrm{~Hz}$ i.e., 500

3. Substrate ITO glass \& silicon wafer

4. Substrate temperature: Room temperature

5. Beam aperture $3.5 \times 0.5 \mathrm{~mm}$

6. Angle of beam w.r.t target $45^{\circ}$

Three variable parameters (laser energy, target to substrate distance and partial pressure of oxygen) were selected for the present experiments. Three levels for each of these parameters were selected for making L9 orthogonal table. These values are shown in Table 2. For each condition as mentioned in orthogonal Table 2, three sets of experiments were carried out. Each thin films of carbon were characterized by Raman spectroscopy (JASCO NRS-1500 W, green laser with wavelength of $514 \mathrm{~nm}$ ), SEM (Hitachi3000S, scanning electron microscope) and TEM (JEOL JEM 3010 , transmission electron microscope) and optical properties were measured by JASCO-V570 UV/VIS/NIR spectrophotometer.

\section{Results and discussion}

\subsection{Band gap measurement by optical method}

The optical band gap is a basic property of the materials. The measured band gap depends not only on the material but also on its characteristics such as crystallinity and stoichiometry. Band gap of the each films were determined by optical method [7]. Optical absorption of all films prepared by conditions mentioned in Table 2 was measured. Assuming the absorption to be equivalent to absorption coefficient for the corresponding wavelength, a graph was plotted between $\{\alpha h v)^{2 / n}$ and $h v$, for two values of $n=1$ for indirect

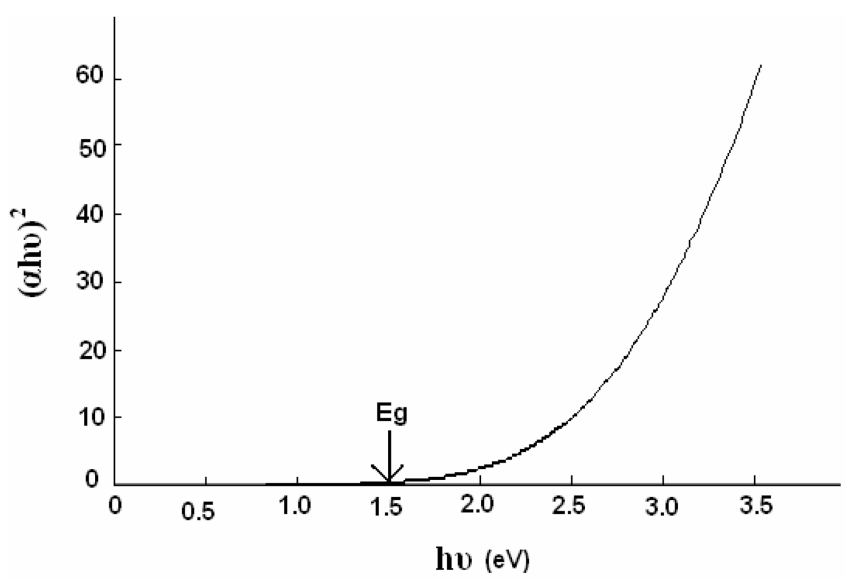

Fig. 3. A typical graph of $(\alpha h v)^{n / 2}$ versus $h v$ when $n=4$ for thin film obtained from L2 experiment. When graph was plotted for $\mathrm{n}=1$, no linear graph was obtained suggested that unlike silicon, carbon films prepared by this method gave only direct band gap.

Table 2. L9 Orthogonal Table Showing Their Combinations Along with Their Three Levels and Optical Band Gap of Each Film

\begin{tabular}{cccccc}
\hline No & Laser energy $(\mathrm{mJ})$ & Distance $(\mathrm{cm})$ & Partial pressure, (Torr) & $\begin{array}{c}\text { Optical gap (direct), (eV) } \\
\text { from three films }\end{array}$ & Mean Band gap (eV) \\
\hline 1 & 160 & 2.5 & $\mathrm{~N}_{2}$ & $1.5,1.6,1.65$ & 1.58 \\
2 & 160 & 3.5 & $\mathrm{O}_{2}, 6 \times 10^{-1}$ & $1.4,1.5,1.5$ & 1.47 \\
3 & 160 & 4.5 & $\mathrm{O}_{2}, 10^{-3}$ & $1.7,1.75,1.8$ & 1.75 \\
4 & 190 & $\mathrm{O}_{2}, 6 \times 10^{-1}$ & $1.7,1.2,1.8$ & 1.57 \\
5 & 190 & $\mathrm{O}_{2}, 10^{-3}$ & $1.9,1.85,1.65$ & 1.80 \\
6 & 190 & 3.5 & $\mathrm{~N}^{-3}$ & $1.7,1.75,1.9$ & 1.78 \\
7 & 220 & 4.5 & $\mathrm{O}_{2}, 10^{-3}$ & $1.8,1.75,1.75$ & 1.77 \\
8 & 220 & 2.5 & $\mathrm{~N}_{2}$ & $1.65,1.7,1.7$ & 1.68 \\
9 & 220 & 3.5 & $\mathrm{O}_{2}, 6 \times 10^{-1}$ & $1.6,1.55,1.55$ & 1.57 \\
\hline
\end{tabular}


band gap and $n=4$ for direct band gap. The graph which gave the linear graph was assumed to be correct. This relationship is based on the equation given below-

$$
\alpha=\left[\mathrm{A}(\mathrm{Eg}-\mathrm{h} v)^{\mathrm{n} / 2}\right] / \mathrm{h} v
$$

The intercept of the linear plot at $\{\alpha h v)^{2 / n}=0$ was taken as magnitude of band gap. A typical graph obtained with carbon thin film prepared under the L2 condition is shown in Fig. 3. In similar fashion band gap for all films were calculated and the results are shown in Table 2. It will be noticed from the result (Table 2), that thin film of carbon obtained from experiments carried out under L2 conditions gave the required band gap of $1.47 \mathrm{eV}$. Hence the amorphous carbon present in the film comprises $\mathrm{sp}^{2} / \mathrm{sp}^{3}$ bonds has an indirect band gap in the range of 0 to $3 \mathrm{eV}$ but it is observed here that the indirect band gap is 0 which depends on $\mathrm{sp}^{2} / \mathrm{sp}^{3}$ ratio [8]. However, Yoshitake et al. [9] also have studied similar thing for ultrananocrystalline diamond/amorphous carbon composite thin films.

\subsection{SEM/TEM studies}

SEM and TEM micrograph of CNTs are shown in Fig. 4 and Fig. 5 synthesized by spray pyrolysis technique. It reveals that the CNTs are nearly uniform in diameter. When thin film of carbon was prepared by PLD, it was necessary to confirm whether the characteristic morphology of CNT has retained its identity. For this purpose SEM of carbon thin film grown on silicon substrate was taken (Fig. 6). This shows that the morphology of CNT has not changed during the PLD deposition except that its diameter has changed from $20 \mathrm{~nm}$ (target material) to $150 \mathrm{~nm}$ (laser deposited film). The reason behind in increasing diameter was unexpected and it is not clear yet. But we think that it might be due to the amorphous carbon surrounded by these

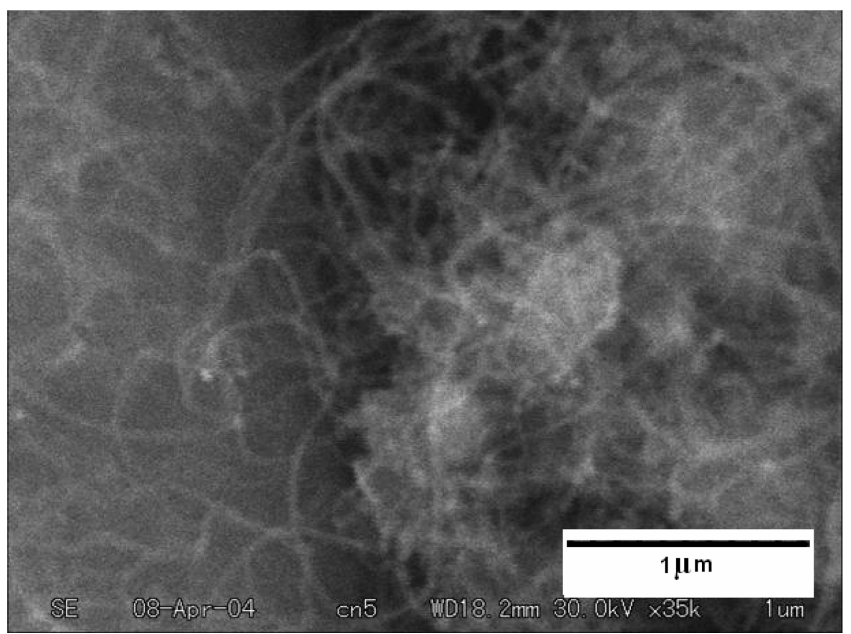

Fig. 4. SEM micrograph of CNT prepared by spray pyrolysis of turpentine oil at $700^{\circ} \mathrm{C}$ using $\mathrm{Fe} / \mathrm{Ni}$ alloy catalyst. The diameter is around $20 \mathrm{~nm}$
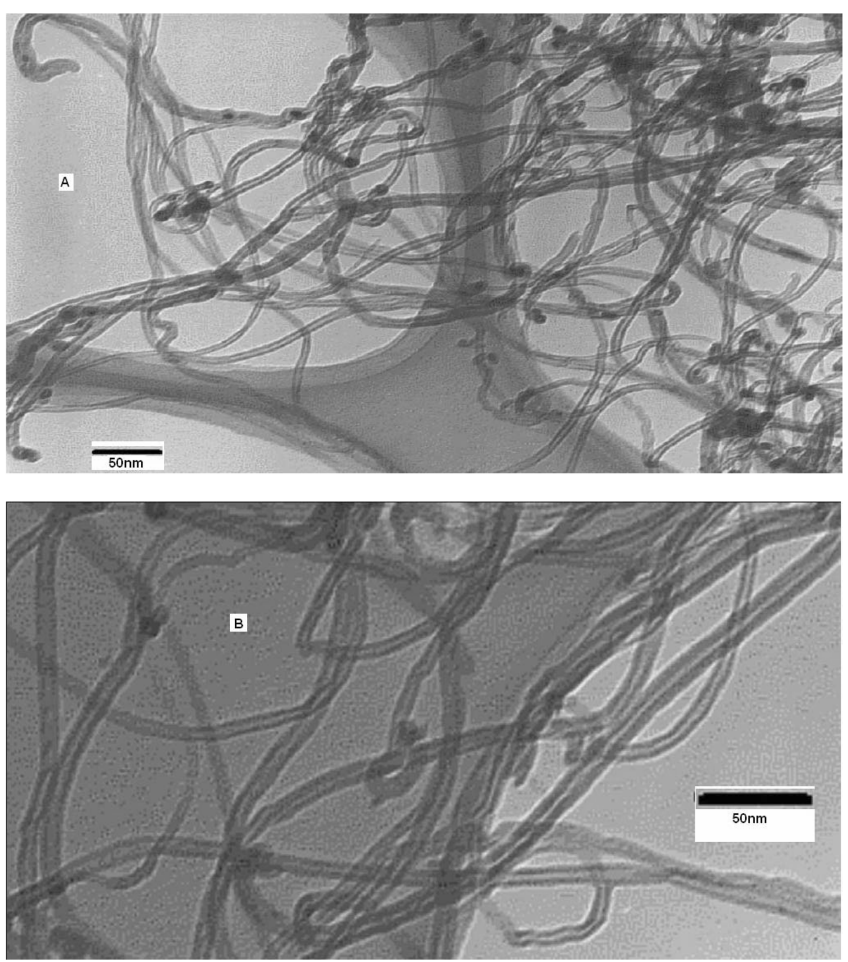

Fig. 5. (A) TEM of CNT as prepared by spray pyrolysis (B) TEM of CNT after purification.

nanotubes. In the state-of-art, during depositing the nanotubes by PLD technique in $\mathrm{O}_{2}$ atmosphere; the amorphous carbon left over on CNTs might be reduce and we will get pure CNTs on the substrates. This attributes that the increase in diameter of the nanotubes due to covering of amorphous carbon on it. It is also confirmed from the Raman spectroscopy studies.

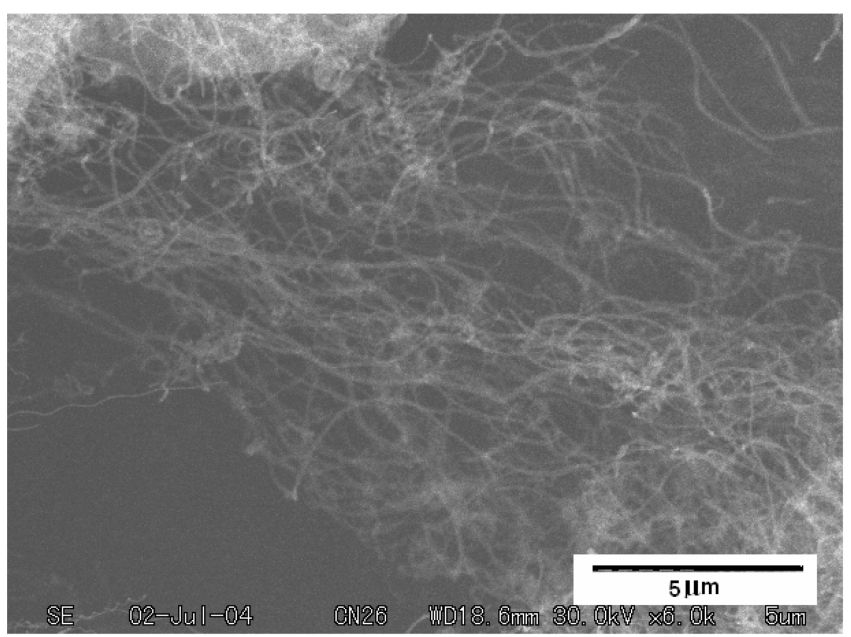

Fig. 6. A typical SEM of the thin film of CNTs on silicon substrate. It is observed that laser ablation of CNT does not spoil its morphology. But the diameter has increased to around $150 \mathrm{~nm}$ from initial $20 \mathrm{~nm}$. 


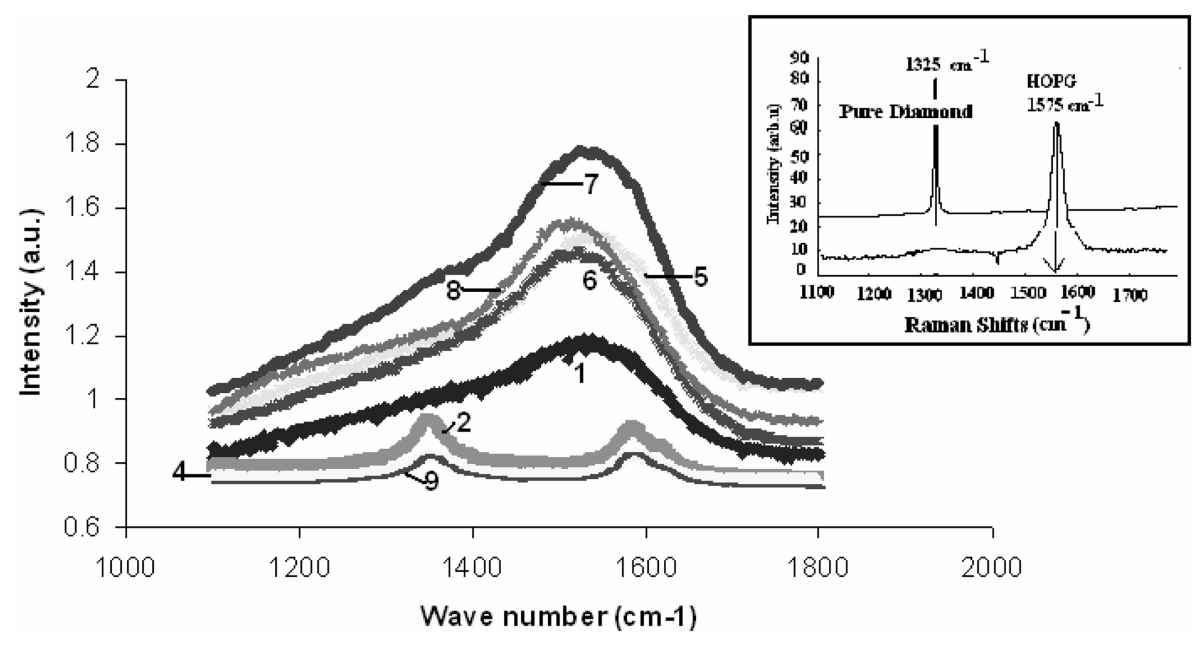

Fig. 7. Raman spectra of carbon thin film prepared under different conditions (as mentioned in Table -1 by laser ablation technique. For comparison Raman spectra of pure diamond and graphite (HOPG) are also shown in the inset.

\subsection{Raman spectroscopy studies}

Raman spectra of all carbon thin film obtained by the spray pyrolysis were taken. Raman spectra obtained with carbon thin films prepared from various parameters (Table1) are shown in Fig. 7. The examination of these spectra suggests that carbon thin films prepared under condition L2, L4 and L9 show the presence of D- and G- bands. It will be noticed from Table- 1 that these films were prepared in the presence of oxygen pressure of $6 \times 10^{-1}$ Torr. Lower oxygen pressure or in presence of nitrogen atmosphere, films contained well defined G-band with tail spectra in the region covering D-band. It is also interesting to note that carbon thin film prepared under L2, L4 and L9 have D-band around $1351 \mathrm{~cm}^{-1}$ (which is very close to D-band obtained with diamond) and G-bands around $1585 \mathrm{~cm}^{-1}$ (which is also very close to G- band for graphite). Whereas carbon films prepared under other conditions showed G-bands in the region of $1528 \mathrm{~cm}^{-1}$ (which is lower than what we get with HOPG) and showing almost no specific peak for D-band or it broadened both G- and D-peaks which means the amorphous carbon has increased in the films and it is also revealed from SEM of the films as mentioned in section 3.2.

It is also noticed from these studies that oxygen pressure in the chambers controls the ratio of $\mathrm{sp}^{2} / \mathrm{sp}^{3}$ such that the carbon material has band gap in the vicinity of $1.5 \mathrm{eV}$ (Fig. 7, L2, L4 and L9). Raman spectra of these films also suggests that for getting carbon thin film with lower band gap, the difference of peak positions between the G-band of HOPG and G-band of carbon materials or D-band of diamond and D-band of carbon materials should be the least. Oxygen pressure in the chamber facilitates the formation of such bands in the carbon thin film.

\section{Conclusions}

Carbon nanotubes of diameter $\sim 20 \mathrm{~nm}$ were synthesized from turpentine oil with $\mathrm{Ni} / \mathrm{Fe}$ catalysts by the spray pyrolysis technique. These carbon nanotubes were used as a target material to deposit thin film of carbon by laser ablation technique. In order to establish suitable condition of getting carbon thin film of band gap around $1.4 \mathrm{eV}$, parameters of laser ablation techniques were varied as given in Table 1. Effect of oxygen pressure in the deposition chamber was selected as one parameter. It is expected that carbon nanotubes might get oxidized due to presence of oxygen during the ablation process resulting into a carbon thin film of required band gap. Band gaps of carbon thin films prepared by laser ablation using the conditions as given in Table 1, were determined by measuring optical absorption and analyzing their data (as discussed in section 3.1). Band gaps of all materials are shown in Table 2. For each condition (Table 1) three samples were prepared and a mean of band gaps obtained from three samples were taken as an accurate value of band gap (Table 2). It is interesting to note that diameter of carbon nanotubes present in the carbon thin film deposited by laser ablation increased from its original diameter of $20 \mathrm{~nm}$ to $150 \mathrm{~nm}$ (Figs. 4-6) with a band gap of $1.47 \mathrm{eV}$.

\section{Acknowledgment}

One of the authors (M.S.) is thankful to the management of Nagoya Institute of Technology, Japan to provide him the Visiting Professorship for 6 months which enabled him to carry out these experiments. 


\section{References}

[1] Sharon M.; Jain S.; Kichambre P.D.; Mukul K., Mater. Chem. and Phys 1998, 56, 284.

[2] Sharon M.; Sundarakoteeswaran N.; Kichambre P.D.; Mukul K.; Ando Y.; Zhao X. Dia. and Relat. Mater 1999, 8, 485.

[3] Sharon M.; Mukhopadhyay I.; Mukhopadhyay K. Sol. Ener. Mate. and Sol. Cells 1997, 45, 35.

[4] Krishna K.M.; Soga T.; Mukhopadhyay K.; Sharon M.;
Umeno M. Sol. Ener. Mater. Sol. Cells 1997, 48, 23.

[5] Mukhopadhyay K.; Mukhopadhyay I.; Sharon M.; Soga T.; and Umeno M. Carbon 1997, 35, 863.

[6] Afre R. A.; Soga T.; Jimbo T.; Mukul K.; Ando Y. and Sharon M.; Somani P.R.; Umeno M. Micro. Mespo. Mate. 2006, 96 (1-3), 184.

[7] Scaife D. E. Solar Energy 1980, 25(1), 41.

[8] Ferrari A.C. and Robertson J. Phys. Rev. B 2000, 61, 14095.

[9] Yoshitake T.; Nagano A.; Itakura M.; Kuwano N.; Hara T.; and Nagayama K. Jpn. J. Appl. Phys. 2007, 46 (38), L936. 